\title{
Experimental study of assistant aids and new nursing method in nursing care work ${ }^{1}$
}

\author{
Nobuyuki Motegi ${ }^{\mathrm{a},{ }^{*}}$, Fumiko Matsuda $^{\mathrm{a}}$, Yuriko Takeuchi ${ }^{\mathrm{a}}$ and Tetsuo Misawa ${ }^{\mathrm{b}}$ \\ anstitute for Science of Labour, Kanagawa,Japan, \\ ${ }^{\mathrm{b}}$ Chiba Institute of Technology, Chiba, JAPAN, \\ motegi-nob@nifty.com
}

\begin{abstract}
This study seeks to evaluate the effect of regular and new nursing methods in nursing care work. Nursing care work often causes low back pain in nursing care worker. The principle of not lifting when transferring patients has been proposed as one way to prevent low back pain. This principle incorporates the use of the patient's strength and assistant aids. A sliding seats and transfer boards have been proposed as assistant aids for the transferring patients. It is necessary to evaluate the effectiveness of these assistant aids in preventing low back pain. Ten subjects performed two tasks in this experiment. Five were nursing experienced persons and five were the inexperienced. EMG results indicated that the new nursing method was less stressful than the methods. A questionnaire revealed that the new method was evaluated more highly than the regular method. Based on these results, we propose that a sliding seats and transfer boards be used in nursing care work.
\end{abstract}

Keywords: assistive devices, electromyography, workload

\section{Introduction}

In Japan, which is already experiencing an aging society, many care workers suffer from work-related musculoskeletal disorders since assistive devices have not been adopted in care-work sites. Nurses and care takers often suffer low back pain when assisting patients. The principle of not lifting when transferring patients has been proposed as one way to prevent low back pain. This principle incorporates the use of the patient's strength and assistant aids. Sliding seats and transfer boards have been proposed as assistant aids for transferring patients. It is necessary to evaluate the effectiveness of these assistant aids in preventing lower back pain. This study seeks to evaluate the effect of regular and new nursing methods in nursing care work.

\section{Method}

Ten subjects performed two tasks in this experiment. Five were nursing experienced persons with a mean age of $61.4 \pm 4.1$ years, and five were inexperienced person with a mean age of $26.2 \pm 8.0$ years. The experienced persons had a mean height of $157.2 \pm 4.3 \mathrm{~cm}$ and a mean weight was $56.0 \pm 5.4 \mathrm{~kg}$. Inexperienced persons had a mean height of $161.0 \pm 1.7 \mathrm{~cm}$ and a mean weight of $55.0 \pm 4.4 \mathrm{~kg}$.

They were all judged to be in good physical health and claimed never to have had any musculoskeletal or cardiovascular problems.

The subjects were evaluated with respect to task time and electromyography (EMG) of the biceps brachii, the trapezius, L4 (low back), and rectus femoris and were asked to complete a questionnaire about tasks. The work examined was transferring from a supine position on the bed to sitting in a wheelchair and a patient from sitting in a wheelchair to supine position on the bed.

The task conditions could be accomplished by two methods (Figure1). The regular nursing method transferred the subject in the usual way. The new nursing method was to transfer the subject using a sliding seat and the transfer boards.

\footnotetext{
${ }^{1}$ Experimental study of assistant aids and new nursing methods in nursing care work.

*Nobuyuki MOTEGI. E-mail: motegi-nob@nifty.com.
} 


\section{Result}

\subsection{Task time}

Mean task times for transfer from a bed to a wheelchair were $140.7 \pm 61.3$ seconds for the regular nurs ing method and $239.4 \pm 61.3$ seconds for the new nursing method. The effect was statistically significant $(\mathrm{p}<0.05)($ Table 1$)$. Mean task time for transfer from a wheelchair to a bed were $129.8 \pm 52.0 \mathrm{sec}-$ onds for the regular nursing method and $186.7 \pm$ 29.6 seconds for the new nursing method. The effect was statistically significant $(\mathrm{p}<0.05)$ (Table 1$)$.
$24.8 \%$ for the regular nursing method and $18.8 \%$ for the new nursing method (Table 2). The mean values of L4 (low back) (left) in \%MVC showed $24.8 \%$ for the regular nursing method and $20.1 \%$ for the new nursing method. The \%MVC at L4 (low back) (left and right) was maximum for the regular nursing method. EMG results thus revealed that the new nursing method had lower loads than the regular nursing method. The effect was statistically significant in all body parts $(\mathrm{p}<0.001)$. The mean \%MVC values for L4 (low back) (right) when transferring from a wheelchair to a bed were $25.5 \%$ for the regular nursing method and $21.8 \%$ for the new nursing

\section{2. $E M G$}

The mean values of L4 (low back) (right) in $\% \mathrm{MVC}$ transfers from a bed to a wheelchair were

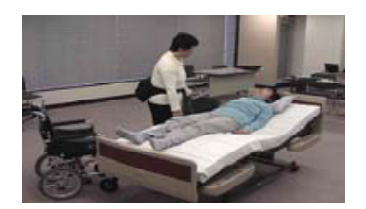

1. Adjustment of bed

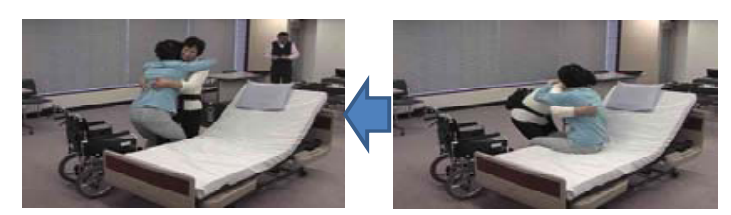

3. Transfer kresent)

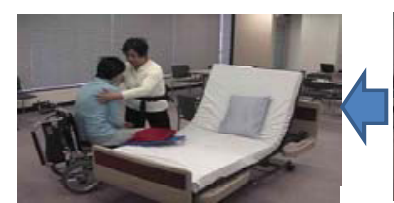

3. Transfer hew)

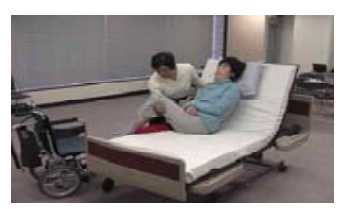

2.Sit position on bed

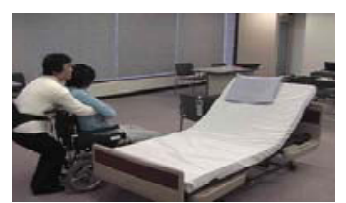

5. Reposition on wheelchair kresent)

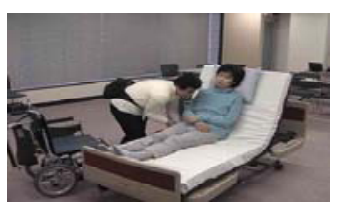

6.Use of sliding seat and transfer board

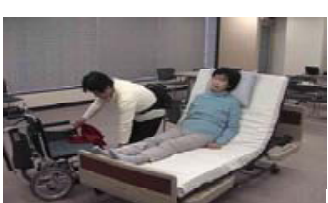

4. Adjustment of wheelchair

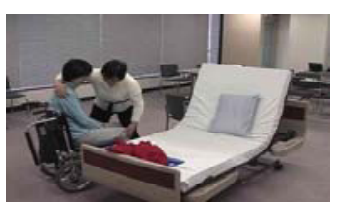

5. Reposition on wheelchair (new)

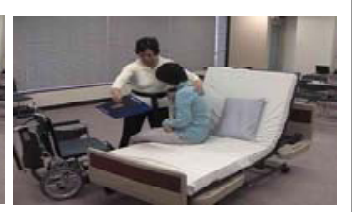

Figure1. Transferring subject from a bed to a wheelchair.

Table 1

Task time (mean and SD)

\begin{tabular}{lccc} 
& \multicolumn{3}{c}{ Transfer from a bed to a wheelchair. } \\
\hline \multicolumn{1}{c}{ Subjects } & Regular mehod & New mehod & t value \\
\hline all subjects & $140.7(61.3)$ & $239.4(96.7)$ & $2.73^{*}$ \\
Experienced subjects & $121.0(37.0)$ & $192.4(39.3)$ & $2.96^{*}$ \\
Inexperienced subjects & $160.4(78.3)$ & $286.4(118.2)$ & 1.99 \\
\hline
\end{tabular}

\begin{tabular}{lllc} 
& \multicolumn{3}{l}{ Transfer from a wheelchair to a bed. } \\
\hline \multicolumn{1}{c}{ Subjects } & Regular mehod & New mehod & t value \\
\hline all subjects & $129.8(52.0)$ & $186.7(29.6)$ & $3.01^{*}$ \\
Experienced subjects & $115.8(37.0)$ & $187.4(40.5)$ & $2.92^{*}$ \\
Inexperienced subjects & $143.8(65.0)$ & $186.0(18.2)$ & 1.40 \\
\hline & & & $*$ \\
& & &
\end{tabular}


Table 2

$\% \mathrm{MVC}$ of EMG (mean and SD)

\begin{tabular}{lllc} 
& \multicolumn{3}{l}{ Transfer from a bed to a wheelchair. } \\
\hline EMG & \multicolumn{2}{l}{ Regular mehod New mehod } & t value \\
\hline Biceps brachii(right) & $20.3(17.5)$ & $14.7(13.7)$ & $10.30^{* * *}$ \\
Biceps brachii(left) & $21.6(19.4)$ & $16.7(13.8)$ & $8.35^{* * *}$ \\
Trapezius(right) & $18.4(17.0)$ & $14.4(13.3)$ & $7.59^{* * *}$ \\
Trapezius(left) & $17.6(15.8)$ & $13.1(11.2)$ & $9.33^{* * *}$ \\
Low back(L4)(right) & $24.8(18.0)$ & $18.8(14.6)$ & $9.90^{* * *}$ \\
Low back(L4)(left) & $24.8(16.4)$ & $20.1(13.2)$ & $7.16^{* * *}$ \\
Rectus femoris(right) & $21.0(18.9)$ & $11.7(9.9)$ & $17.06^{* * *}$ \\
Rectus femoris(left) & $19.1(18.4)$ & $10.3(10.0)$ & $16.67^{* * *}$ \\
\hline
\end{tabular}

\begin{tabular}{llcc} 
& \multicolumn{3}{l}{ Transfer from a wheelchair to a bed. } \\
\hline EMG & \multicolumn{4}{l}{ Regular mehod New mehod } & t value \\
\hline Biceps brachii(right) & $23.5(20.4)$ & $15.1(14.9)$ & $12.64^{* * *}$ \\
Biceps brachii(left) & $24.7(20.5)$ & $15.9(14.2)$ & $13.36^{* * *}$ \\
Trapezius(right) & $20.6(18.6)$ & $15.5(14.4)$ & $8.24 * * *$ \\
Trapezius(left) & $18.3(16.4)$ & $14.0(11.4)$ & $8.14^{* * *}$ \\
Low back(L4)(right) & $25.5(17.1)$ & $21.8(15.2)$ & $5.73^{* * *}$ \\
Low back(L4)(left) & $29.6(19.6)$ & $22.4(15.7)$ & $8.67 * * *$ \\
Rectus femoris(right) & $17.4(14.7)$ & $12.2(10.9)$ & $10.71 * * *$ \\
Rectus femoris(left) & $16.6(15.3)$ & $9.9(10.2)$ & $13.79 * * *$ \\
\hline
\end{tabular}

Table 3

Questionnaire on tasks for subjects

\begin{tabular}{lccc}
\multicolumn{1}{c}{ Transfer of tasks } & & & \\
\hline & Good & Neutral & Bad \\
\hline Regular mehod & 0 & 6 & 4 \\
New mehod & 6 & 4 & 0 \\
\hline
\end{tabular}

\begin{tabular}{lccc}
\multicolumn{1}{c}{ Workload of tasks } & \multicolumn{3}{c}{} \\
\hline & Yes & Both either & No \\
\hline Regular mehod & 6 & 1 & 3 \\
New mehod & 0 & 3 & 7 \\
\hline
\end{tabular}

method (Table 2). The mean values for L4 (low back) (left) in \%MVC were $29.6 \%$ for the regular nursing method and $22.4 \%$ for the new nursing method (Table 2). L4 (low back) (left) exhibited maximum \%MVC for the regular nursing method. EMG results indicated that the loads in the new nursing method were less stressful than those in the regular method. The effect was statistically significant in all body parts $(p<0.001)$.

\subsection{Questionnaire on tasks}

The results of questionnaire about transfer tasks indicated that the new nursing method was evaluated more highly than the regular nursing method (Table 3 ). The effect was statistically significant $(\mathrm{p}<0.01)$.

The results of a questionnaire about workloads indicated that the new nursing method was evaluated more highly than the regular nursing method (Table 3 ). The effect was statistically significant $(p<0.05)$.

\section{Discussion}

This study sought to evaluate the effect of regular and new nursing methods in nursing care work.

Task time analysis results indicated that the new nursing method took longer than the regular nursing method because the new nursing method employed assistant aids for the transfer (Table 1).

EMG results indicated that loads in the new nursing method were less than those in the regular nursing method (Table 2). The effect was statistically significant in all body parts $(\mathrm{p}<0.001)$. We observed that the new nursing method, which employed assistant aids muscle loads.

Questionnaire results indicated that the new nursing method was evaluated more highly than the regular nursing method (Table 3). Experienced persons evaluated the new nursing method as being especially good. Patients commented that they could not detect any difference in comfort between the new nursing method and the regular nursing method.

Based on these results, we propose that a sliding seats and transfer boards be used in nursing care work.

\section{References}

[1] Kimiko Tomioka,Yumi Higuchi, and Hidemi Shindo, A Validation study of devices designed to reduce loads in provision of care: Whether these assistive devices are used, or not, and whether work posture is appropriate or not, San Ei Shi 49(2007),113-121

[2] A. Grag, B. Owen, D. Beller and J. Banaag, A biomechanical and ergonomic evaluation of patient transferring tasks: bed to wheelchair and wheelchair to bed, Ergonomics, 1991, vol34, No3, 289-312.

[3] A. Grag, B. Owen, D. Beller and J. Banaag, A biomechanical and ergonomic evaluation of patient transferring tasks: wheelchair to shower chair and shower chair to wheelchair, ERGONOMICS, 1991, vol34, No4, 407-419. 